JOURNAL OF INTEGRAL EQUATIONS

AND APPLICATIONS

Volume 12, Number 1, Spring 2000

\title{
ON THE FREDHOLM INDICES OF ASSOCIATED SYSTEMS OF WIENER-HOPF EQUATIONS
}

\author{
A. BÖTTCHER, S.M. GRUDSKY AND I.M. SPITKOVSKY
}

\begin{abstract}
Every matrix function $A \in L_{n \times n}^{\infty}(\mathbf{R})$ generates a Wiener-Hopf integral operator on $L_{n}^{2}\left(\mathbf{R}_{+}\right)$, the direct sum of $n$ copies of $L^{2}\left(\mathbf{R}_{+}\right)$. The associated Wiener-Hopf integral operator is the operator $W(\tilde{A})$ where $\tilde{A}(x):=A(-x)$. We discuss the connection between the Fredholm indices Ind $W(A)$ and Ind $W(\tilde{A})$. Our main result says that if $A$ has at most a finite number $d$ of discontinuities on $\mathbf{R} \cup\{\infty\}$ and both $W(A)$ and $W(\tilde{A})$ are Fredholm, then
\end{abstract}

$$
|\operatorname{Ind} W(A)+\operatorname{Ind} W(\tilde{A})| \leq d(n-1) ;
$$

conversely, given integers $\kappa$ and $\nu$ satisfying $|\kappa+\nu| \leq d(n-1)$, there exist $A \in L_{n \times n}^{\infty}(\mathbf{R})$ with at most $d$ discontinuities such that $W(A)$ is Fredholm of index $\kappa$ and $W(\tilde{A})$ is Fredholm of index $\nu$.

1. Introduction and main results. Given a measurable subset $\Omega$ of the real line $\mathbf{R}$, we denote by $L_{n \times n}^{p}(\Omega)$ and $L_{n}^{p}(\Omega)$ the $n \times n$ matrix functions with entries in $L^{p}(\Omega)$ and the column vectors of height $n$ with components in $L^{p}(\Omega)$, respectively. For $A \in L_{n \times n}^{\infty}(\mathbf{R})$, the convolution operator with the symbol $A$ is the operator

$$
C(A): L_{n}^{2}(\mathbf{R}) \longrightarrow L_{n}^{2}(\mathbf{R}), \quad f \longmapsto \mathcal{F}^{-1} A \mathcal{F} f
$$

where $\mathcal{F}$ is the Fourier transform,

$$
(\mathcal{F} f)(x):=\int_{\mathbf{R}} f(t) e^{i x t} d t, \quad x \in \mathbf{R} .
$$

Let $\mathbf{R}_{+}=(0, \infty)$. The compression of $C(A)$ to $L_{n}^{2}\left(\mathbf{R}_{+}\right)$is referred to as the Wiener-Hopf operator with the symbol $A$ and will be denoted by $W(A)$. Thus,

$$
W(A): L_{n}^{2}\left(\mathbf{R}_{+}\right) \longrightarrow L_{n}^{2}\left(\mathbf{R}_{+}\right), \quad f \longmapsto P \mathcal{F}^{-1} A \mathcal{F} f,
$$

Received by the editors in revised form on June 10, 1999.

Copyright (C)2000 Rocky Mountain Mathematics Consortium 
$P$ being the orthogonal projection of $L_{n}^{2}(\mathbf{R})$ onto $L_{n}^{2}\left(\mathbf{R}_{+}\right)$. For $A$ in $L_{n \times n}^{\infty}(\mathbf{R})$, we define $\tilde{A} \in L_{n \times n}^{\infty}(\mathbf{R})$ by

$$
\tilde{A}(x):=A(-x), \quad x \in \mathbf{R} .
$$

The Wiener-Hopf operator $W(\tilde{A})$ is called the associated operator of $W(A)$. A moment's thought reveals that $W(\tilde{A})$ is unitarily equivalent to the compression of $C(A)$ to $L_{n}^{2}\left(\mathbf{R}_{-}\right)$, where $\mathbf{R}_{-}=(-\infty, 0)$.

If $A$ is the Fourier transform of an $L^{1}$ matrix function, $A=\mathcal{F} k$ with $k \in L_{n \times n}^{1}(\mathbf{R})$, then $W(A)$ and $W(\tilde{A})$ can be written in the form

$$
\begin{aligned}
& (W(A) f)(t)=\int_{0}^{\infty} k(t-s) f(s) d s, \quad t>0, \\
& (W(\tilde{A}) f)(t)=\int_{0}^{\infty} k(s-t) f(s) d s, \quad t>0 .
\end{aligned}
$$

The symbol $A(x)=-\operatorname{sign} x(n=1)$ induces the Cauchy singular integral operator on $\mathbf{R}_{+}$,

$$
(W(A) f)(t)=\frac{1}{\pi i} \int_{0}^{\infty} \frac{f(s)}{s-t} d s, \quad t>0,
$$

and in the corresponding formula for $W(\tilde{A})$ we have to replace $s-t$ by $t-s$. Finally, if $A(x)=e^{i x}(n=1)$, then

$$
\begin{aligned}
& (W(A) f)(t)= \begin{cases}f(t-1) & \text { for } t>1, \\
0 & \text { for } 0<t<1,\end{cases} \\
& (W(\tilde{A}) f)(t)=f(t+1) \text { for } t>0 .
\end{aligned}
$$

It is well known that the answers to many questions on the operator $W(A)$ depend not only on the properties of $W(A)$ itself but also on the properties of the associated operator $W(\tilde{A})$. This is, for instance, the case when studying the finite section method for $W(A)$, see $[\mathbf{4}, \mathbf{6}]$ or the Fredholm determinants of the truncations of $W(A)$, see [18]. Moreover, questions on the connection between left and right WienerHopf factorizations of the matrix function $A$ are always questions on the relation between certain properties of $W(A)$ and $W(\tilde{A})$, see $[\mathbf{5}, \mathbf{1 1}]$. 
Recall that a bounded linear operator $T: H \rightarrow H$ is said to be Fredholm if its range $\operatorname{Im} T$ is closed and the dimensions of the kernel $\operatorname{Ker} T:=\{f \in H: T f=0\}$ and the cokernel Coker $T:=H / \operatorname{Im} T$ are finite. In that case the Fredholm index of $T$ is

$$
\operatorname{Ind} T:=\operatorname{dim} \operatorname{Ker} T-\operatorname{dim} \operatorname{Coker} T .
$$

In the scalar case $(n=1), W(\tilde{A})$ is the transpose operator of $W(A)$ and therefore $W(A)$ is Fredholm of index $\kappa$ if and only if $W(\tilde{A})$ is Fredholm of index $-\kappa$. This paper concerns the connection between the Fredholm indices of $W(A)$ and $W(\tilde{A})$ in the matrix case, $n \geq 2$. To be more precise, we consider the following questions:

(a) Does the Fredholmness of $W(A)$ imply that $W(\tilde{A})$ is also Fredholm?

(b) If $W(A)$ and $W(\tilde{A})$ are Fredholm, does it follow that Ind $W(A)$ is equal to $-\operatorname{Ind} W(\tilde{A})$ ?

The answers to both questions are known to be in the affirmative in case $A$ belongs to certain classes of symbols, for example, if

$$
A \in\left[C(\dot{\mathbf{R}})+H_{+}^{\infty}(\mathbf{R})\right]_{n \times n} \cup\left[C(\dot{\mathbf{R}})+H_{-}^{\infty}(\mathbf{R})\right]_{n \times n} \cup P Q C_{n \times n},
$$

see $[\mathbf{4}, \mathbf{5}, \mathbf{1 1}]$. Here $C(\dot{\mathbf{R}})$ stands for the continuous functions on $\mathbf{R}$ with finite and equal limits at $\pm \infty, H_{ \pm}^{\infty}(\mathbf{R})$ is the algebra of the (nontangential) boundary values of analytic and bounded functions in $\mathbf{C}_{ \pm}:=\{z \in \mathbf{C}: \pm \operatorname{Im} z>0\}$ and $P Q C$ denotes the piecewise quasicontinuous functions.

We will show that the two questions nevertheless have negative answers for general $A \in L_{n \times n}^{\infty}(\mathbf{R}), n \geq 2$.

Let $A P$ be the $C^{*}$-algebra of all continuous almost periodic functions on $\mathbf{R}$, that is, the smallest closed subalgebra of $L^{\infty}(\mathbf{R})$ which contains the set $\cup_{\lambda \in \mathbf{R}}\left\{e_{\lambda}\right\}$ where $e_{\lambda}(x):=e^{i \lambda x}$.

Proposition 1.1. There exist $A \in A P_{2 \times 2}$ such that $W(A)$ is invertible but $W(\tilde{A})$ is not Fredholm.

Thus the answer to question (a) is negative within $A P_{n \times n}, n \geq 2$. If $F \in A P_{n \times n}$ and $W(F)$ is Fredholm, then $W(F)$ is automatically 
invertible, see [9] and [10]. Hence, for $A \in A P_{n \times n}$ the answer to question (b) remains positive.

It is well known that much evil with Wiener-Hopf operators begins with semi-almost periodic symbols, see, for example, [3, p. 61], that is, with symbols in the smallest closed subalgebra $S A P$ of $L^{\infty}(\mathbf{R})$ which contains $A P \cup C(\overline{\mathbf{R}})$, where $C(\overline{\mathbf{R}})$ stands for the continuous functions on $\mathbf{R}$ which have finite limits at $-\infty$ and $+\infty$. This experience is supported by the following result, which shows that the answer to question (b) is already negative within $S A P_{n \times n}, n \geq 2$.

Proposition 1.2. There are $A \in S A P_{2 \times 2}$ such that $W(A)$ is Fredholm of index 0 and $W(\tilde{A})$ is Fredholm of index 1.

This proposition suggests to sharpen question (b) as follows:

(c) Given two integers $\kappa$ and $\nu$, is there a matrix function $A$ such that $W(A)$ is Fredholm of index $\kappa$ and $W(\tilde{A})$ is Fredholm of index $\nu$ ?

An answer is given by the following theorem, which is the main result of this paper. Notice that $A \in L_{n \times n}^{\infty}(\mathbf{R})$ is said to have at most $k$ discontinuities on $\mathbf{R} \cup\{\infty\}$ if there are $x_{1}, \ldots, x_{k} \in \mathbf{R} \cup\{\infty\}$ such that $A$ is continuous on $(\mathbf{R} \cup\{\infty\}) \backslash\left\{x_{1}, \ldots, x_{k}\right\}$. We call a point $x_{0} \in \mathbf{R} \cup\{\infty\}$ a harmless discontinuity of $A$ if the matrix function $A$ is discontinuous at $x_{0}$ and if there exist an open neighborhood $U$ of $x_{0}$ and a matrix function

$$
\begin{aligned}
B \in\left[C(\dot{\mathbf{R}})+H_{+}^{\infty}(\mathbf{R})\right]_{n \times n} & \cup\left[C(\dot{\mathbf{R}})+H_{-}^{\infty}(\mathbf{R})\right]_{n \times n} \\
& \cup P Q C_{n \times n} \cup A P_{n \times n}
\end{aligned}
$$

such that $B$ is continuous on $U \backslash\left\{x_{0}\right\}$ and $A|U=B| U$. A discontinuity which is not harmless will be called a severe discontinuity.

Theorem 1.3. If $A \in L_{n \times n}^{\infty}(\mathbf{R})$ has at most a finite number $m$ of harmless discontinuities and at most a finite number $d$ of severe discontinuities on $\mathbf{R} \cup\{\infty\}$ and both $W(A)$ and $W(\tilde{A})$ are Fredholm, then

$$
|\operatorname{Ind} W(A)+\operatorname{Ind} W(\tilde{A})| \leq d(n-1) .
$$

Conversely, given integers $\kappa$ and $\nu$ satisfying $|\kappa+\nu| \leq d(n-1)$, there exist $A \in L_{n \times n}^{\infty}(\mathbf{R})$ with at most d severe discontinuities and no other 
discontinuities on $\mathbf{R} \cup\{\infty\}$ such that $W(A)$ is Fredholm of index $\kappa$ and $W(\tilde{A})$ is Fredholm of index $\nu$.

Here is an obvious consequence of Theorem 1.3.

Corollary 1.4. Given any natural number $n \geq 2$ and any two integers $\kappa$ and $\nu$, there exist $A \in L_{n \times n}^{\infty}(\mathbf{R})$ with at most finitely many discontinuities such that $W(A)$ is Fredholm of index $\kappa$ and $W(\tilde{A})$ is Fredholm of index $\nu$.

As functions in $S A P$ have at most a discontinuity at $\infty$, we also arrive at the following straightforward consequence of Theorem 1.3, which shows that Theorem 1.2 cannot be "improved."

Corollary 1.5. There is no $A \in S A P_{2 \times 2}$ such that $W(A)$ is Fredholm of index 0 and $W(\tilde{A})$ is Fredholm of index 2 .

Of course, Theorem 1.3 even implies that if $A \in S A P_{n \times n}, W(A)$ is Fredholm of index $\kappa$ and $W(\tilde{A})$ is Fredholm of index $\nu$, then necessarily $|\kappa+\nu| \leq n-1$. This leads to the following question:

(d) Given integers $\kappa$ and $\nu$ satisfying $|\kappa+\nu| \leq n-1$, is there a matrix function $A \in S A P_{n \times n}$ such that $W(A)$ is Fredholm of index $\kappa$ and $W(\tilde{A})$ is Fredholm of index $\nu$ ?

The answer is yes.

Theorem 1.6. If $\kappa$ and $\nu$ are any integers subject to the condition $|\kappa+\nu| \leq n-1$, then there exist $A \in S A P_{n \times n}$ such that $W(A)$ is Fredholm of index $\kappa$ and $W(\tilde{A})$ is Fredholm of index $\nu$.

The rest of the paper is devoted to the proofs of the results quoted above. The simple proof of Proposition 1.1 is in Section 2. To prove Theorem 1.3, we will have recourse to Wiener-Hopf factorization, and in this context passage from the real line to the unit circle makes things more comfortable. In Section 3 we therefore translate Theorem 1.3 into the language of Toeplitz operators, and then we give a proof of the 
Toeplitz analogue of the first assertion of Theorem 1.3.

The paper contains two proofs of the second part of Theorem 1.3. The first of these proofs is given in Section 4. The approach of Section 4 is based on the well-known Fredholm criterion for Wiener-Hopf operators with piecewise continuous symbols. Proposition 4.4 in conjunction with a few standard arguments implies the second part of Theorem 1.3. This proposition does even more: it shows that we can find symbols $A$ which are analytic in the plane minus a finite number of points such that $W(A)$ and $W(\tilde{A})$ have prescribed indices. In other words, the symbols we are looking for are in fact not as exotic as one might expect at the first glance.

In Section 5 we first cite a Fredholm criterion and an index formula for Wiener-Hopf operators with "Wiener" $S A P$ symbols. This result is then used to prove Theorem 1.6. Clearly, Theorem 1.6 and standard localization techniques yield a second proof of the second assertion of Theorem 1.3. Notice, however, that the machinery employed in Section 5 is much heavier than the tools we are using in Section 4. Finally, Proposition 1.2 is an obvious consequence of Theorem 1.6.

2. Almost periodic symbols. This short section contains the proof of Proposition 1.1. We remark that this proposition is in fact a triviality and that it is certainly known to specialists. However, we have not found it explicitly in the literature, although it is already noted in $\left[\mathbf{1 6}\right.$, p. 1736] that there are $A \in L_{2 \times 2}^{\infty}(\mathbf{R})$ such that $W(A)$ is invertible and $W(\tilde{A})$ is not Fredholm.

It is well known and easily seen that

$$
W\left(F_{-} G F_{+}\right)=W\left(F_{-}\right) W(G) W\left(F_{+}\right)
$$

whenever $F_{ \pm} \in\left[H_{ \pm}^{\infty}(\mathbf{R})\right]_{n \times n}$ and $G \in L_{n \times n}^{\infty}(\mathbf{R})$. The collection of all $F_{ \pm} \in\left[H_{ \pm}^{\infty}(\mathbf{R})\right]_{n \times n}$ for which $F_{ \pm}^{-1}$ also belongs to $\left[H_{ \pm}^{\infty}(\mathbf{R})\right]_{n \times n}$ will be denoted by $G\left[H_{ \pm}^{\infty}(\mathbf{R})\right]_{n \times n}$. From (2.1) we infer in particular that $W\left(F_{ \pm}\right)$is invertible if $F_{ \pm} \in G\left[H_{ \pm}^{\infty}(\mathbf{R})\right]_{n \times n}$.

Proof of Proposition 1.1. Put

$$
A(x)=\left(\begin{array}{cc}
e^{i x} & 1 \\
0 & e^{-i x}
\end{array}\right) .
$$


Then $A=A_{-} A_{+}$with

$$
A_{-}(x)=\left(\begin{array}{cc}
1 & 0 \\
e^{-i x} & -1
\end{array}\right), \quad A_{+}(x)=\left(\begin{array}{cc}
e^{i x} & 1 \\
1 & 0
\end{array}\right),
$$

and since $A_{ \pm} \in G\left[H_{ \pm}^{\infty}(\mathbf{R})\right]_{2 \times 2}$, we see that $W(A)$ is invertible. On the other hand, $\tilde{A}=H_{-} D H_{+}$with

$$
\begin{aligned}
H_{-}(x) & =\left(\begin{array}{ll}
1 & 0 \\
0 & 1
\end{array}\right), \\
D(x) & =\left(\begin{array}{cc}
e^{-i x} & 0 \\
0 & e^{i x}
\end{array}\right), \\
H_{+}(x) & =\left(\begin{array}{cc}
1 & e^{i x} \\
0 & 1
\end{array}\right) .
\end{aligned}
$$

As $H_{ \pm} \in G\left[H_{ \pm}^{\infty}(\mathbf{R})\right]_{2 \times 2}$, the Fredholmness of $W(\tilde{A})$ would imply that $W(D)$ is Fredholm. However, from representations (1.1) and (1.2) we infer that $W(D)$ has infinite kernel and cokernel dimensions.

3. Toeplitz operators and Wiener-Hopf factorization. Our proofs of Theorems 1.3 and 1.6 make use of Wiener-Hopf factorization, and therefore it is convenient to pass from the real line $\mathbf{R}$ to the complex unit circle $\mathbf{T}$ or, equivalently, from Wiener-Hopf integral operators to Toeplitz operators.

Let $G$ be a matrix function in $L_{n \times n}^{\infty}(\mathbf{T})$. Denote by $G_{k} \in \mathbf{C}^{n \times n}$, $k \in \mathbf{Z}$, the Fourier coefficients of $G$. Further, let $l^{2}\left(\mathbf{Z}_{+}, \mathbf{C}^{n}\right)$ stand for the $\mathbf{C}^{n}$-valued $l^{2}$ space over $\mathbf{Z}_{+}:=\{0,1,2, \ldots\}$. The operator induced on $l^{2}\left(\mathbf{Z}_{+}, \mathbf{C}^{n}\right)$ by the matrix

$$
\left(\begin{array}{cccc}
G_{0} & G_{-1} & G_{-2} & \cdots \\
G_{1} & G_{0} & G_{-1} & \cdots \\
G_{2} & G_{1} & G_{0} & \cdots \\
\cdots & \cdots & \cdots & \cdots
\end{array}\right)
$$

is called the Toeplitz operator with the symbol $G$ and is denoted by $T(G)$.

Given $A \in L_{n \times n}^{\infty}(\mathbf{R})$, define $A^{\#} \in L_{n \times n}^{\infty}(\mathbf{T})$ by

$$
A^{\#}(t)=A\left(i \frac{1+t}{1-t}\right), \quad t \in \mathbf{T} \backslash\{1\} .
$$


Rosenblum and Devinatz were probably the first to observe that there exists an isometric isomorphism $U$ of $L_{n}^{2}\left(\mathbf{R}_{+}\right)$onto $l^{2}\left(\mathbf{Z}_{+}, \mathbf{C}^{n}\right)$ such that

$$
W(A)=U^{-1} T\left(A^{\#}\right) U
$$

see, e.g., [4, p. 400]. This implies the following.

Lemma 3.1. The Wiener-Hopf integral operator $W(A)$ is Fredholm of index $\kappa$ if and only if the Toeplitz operator $T\left(A^{\#}\right)$ is Fredholm of index $\kappa$.

If $A \in L_{n \times n}^{\infty}(\mathbf{R})$, we have $(\tilde{A})^{\#}(t)=A^{\#}(1 / t)$ for $t \in \mathbf{T}$. Thus, the associated operator of the Toeplitz operator $T(G)$ is defined as $T(\tilde{G})$ with $\tilde{G}(t):=G(1 / t), t \in \mathbf{T}$. Notice that if $T(G)$ is given by the matrix $\left(G_{j-k}\right)_{j, k=0}^{\infty}$, then $T(\tilde{G})$ has the matrix $\left(G_{k-j}\right)_{j, k=0}^{\infty}$.

The following two theorems summarize a few basic and well-known properties of Toeplitz operators. Theorem 3.2 (a) is due to Gohberg and Krein, and Theorem 3.2 (c) is a version of the so-called local principle of Simonenko. Theorem 3.3 is known from the work of Gohberg, Krupnik, Douglas, Sarason, Coburn, Feldman and Karlovich. Full proofs are in $[4,5,11]$.

Theorem 3.2. (a) If $G \in[C(\mathbf{T})]_{n \times n}$, then $T(G)$ is Fredholm if and only if $\operatorname{det} G$ has no zeros on $\mathbf{T}$. In that case, Ind $T(G)$ is minus the winding number of $\operatorname{det} G$ about the origin.

(b) If $G, H \in L_{n \times n}^{\infty}(\mathbf{T})$ and if at each point of $\mathbf{T}$ at least one of the matrix functions $G$ and $H$ is continuous, then $T(G H)-T(G) T(H)$ is a compact operator.

(c) Let $G \in L_{n \times n}^{\infty}(\mathbf{T})$ and suppose for each $\tau \in \mathbf{T}$ there exist an open subarc $\Gamma_{\tau} \subset \mathbf{T}$ containing $\tau$ and a matrix function $G_{\tau} \in L_{n \times n}^{\infty}(\mathbf{T})$ such that $G=G_{\tau}$ on $\Gamma_{\tau}$ and $T\left(G_{\tau}\right)$ is Fredholm. Then $T(G)$ is also Fredholm.

A matrix function $G \in L_{n \times n}^{\infty}(\mathbf{T})$ is said to have $m$ harmless and $d$ severe discontinuities on $\mathbf{T}$ if $G=A^{\#}$ where $A$ has $m$ harmless and $d$ severe discontinuities on $\mathbf{R} \cup\{\infty\}$. 
Theorem 3.3. If $G \in L_{n \times n}^{\infty}(\mathbf{T})$ has at most finitely many harmless discontinuities and both $T(G)$ and $T(\tilde{G})$ are Fredholm, then $\operatorname{Ind} T(G)=$ $-\operatorname{Ind} T(\tilde{G})$.

Let $L^{p}(\mathbf{T}), 0<p<\infty$, be the usual Lebesgue space of complex valued functions. Put

$$
\begin{aligned}
& \mathbf{D}_{+}:=\{z \in \mathbf{C}:|z|<1\}, \\
& \mathbf{D}_{-}:=\{z \in \mathbf{C}:|z|>1\} \cup\{\infty\} .
\end{aligned}
$$

The Hardy space $H_{+}^{p}(\mathbf{T}), 0<p<\infty$, is defined as the set of all analytic functions $f$ in $\mathbf{D}_{ \pm}$for which

$$
\|f\|_{p}^{p}:=\sup _{0<r^{ \pm 1}<1} \frac{1}{2 \pi} \int_{0}^{2 \pi}\left|f\left(r e^{i \theta}\right)\right|^{p} d \theta<\infty .
$$

We remark that $\|\cdot\|_{p}$ is a norm for $p \geq 1$. As usual, we identify functions in $H_{ \pm}^{p}(\mathbf{T})$ with their (nontangential) boundary values on $\mathbf{T}$. We denote by $G\left[H_{ \pm}^{p}(\mathbf{T})\right]_{n \times n}$ the matrix functions $F_{ \pm}$in $\left[H_{ \pm}^{p}(\mathbf{T})\right]_{n \times n}$ for which $F_{ \pm}^{-1}$ also belongs to $\left[H_{ \pm}^{p}(\mathbf{T})\right]_{n \times n}$.

Let $\mathcal{R}$ be the rational functions without poles on $\mathbf{T}$. We think of $\mathcal{R}$ as a subset of $L^{2}(\mathbf{T})$. For $f \in \mathcal{R}$, the Cauchy singular integral

$$
(S f)(t):=\frac{1}{\pi i} \int_{\mathbf{T}} \frac{f(\tau)}{\tau-t} d \tau, \quad t \in \mathbf{T}
$$

exists in the principal value sense, and it is well known that $S$ extends to a bounded operator on $L^{2}(\mathbf{T})$.

A right Wiener-Hopf factorization in $L^{2}(\mathbf{T})$ of a matrix function $G \in L_{n \times n}^{\infty}(\mathbf{T})$ is a representation

$$
G(t)=G_{-}(t) M(t) G_{+}(t), \quad M(t)=\operatorname{diag}\left(t^{\varrho_{1}}, \ldots, t^{\varrho_{n}}\right)
$$

for almost all $t \in \mathbf{T}$ such that $\varrho_{1}, \ldots, \varrho_{n}$ are integers, $G_{ \pm}$are in $G\left[H_{ \pm}^{2}(\mathbf{T})\right]_{n \times n}$ and the operator $G_{+}^{-1} S G_{+} I$ is bounded on $L_{n}^{2}(\mathbf{T})$. Notice that if $f \in \mathcal{R}_{n}$, then $G_{+}^{-1} S G_{+} f$ is a well-defined element of $L_{n}^{1}(\mathbf{T})$. That $G_{+}^{-1} S G_{+} I$ is bounded on $L_{n}^{2}(\mathbf{T})$ means that the map $f \mapsto G_{+}^{-1} S G_{+} f$ is actually a map of $\mathcal{R}_{n}$ into $L_{n}^{2}(\mathbf{T})$ which extends 
to a bounded linear operator of $L_{n}^{2}(\mathbf{T})$ into itself. In what follows we abbreviate "right Wiener-Hopf factorization in $L^{2}(\mathbf{T})$ " simply to " $L^{2}$ factorization." The sum $\varrho_{1}+\cdots+\varrho_{n}$ is referred to as the total index of the given $L^{2}$ factorization.

The following theorem goes back to Gohberg and Krein [7] and to Simonenko $[\mathbf{1 3}, \mathbf{1 4}, \mathbf{1 5}]$. Full proofs are also in $[\mathbf{5}]$ and $[\mathbf{1 1}]$.

Theorem 3.4. Let $G \in L_{n \times n}^{\infty}(\mathbf{T})$. The operator $T(G)$ is Fredholm of index $\kappa$ if and only if $G$ is invertible in $L_{n \times n}^{\infty}(\mathbf{T})$ and $G$ has an $L^{2}$ factorization with the total index $-\kappa$.

The next two results provide us with refinements of Theorem 3.4. These results are also well known, see, e.g., [17], and we give proofs only for the reader's convenience.

Proposition 3.5. Let $G$ be a matrix function in $L_{n \times n}^{\infty}(\mathbf{T})$ and suppose that $G$ extends to an analytic matrix function in some open set $U \subset \mathbf{C}$ such that $U \cap \mathbf{T} \neq \varnothing$. If $G=G_{-} M G_{+}$is an $L^{2}$ factorization, then $G_{ \pm}$and $G_{ \pm}^{-1}$ are analytic in $U \backslash\{0\}$.

Proof. We have $G_{+}=M^{-1} G_{-}^{-1} G$ almost everywhere on $\mathbf{T}$. Define

$$
\Psi(z):= \begin{cases}G_{+}(z) & \text { for } z \in\left(\mathbf{D}_{+} \cup \mathbf{T}\right) \cap U, \\ M^{-1}(z) G_{-}^{-1}(z) G(z) & \text { for } z \in\left(\mathbf{D}_{-} \cup \mathbf{T}\right) \cap U .\end{cases}
$$

The assertion will follow once we have shown that $\Psi$ is analytic in $U \backslash\{0\}$.

To show that $\Psi$ is analytic in $U \backslash\{0\}$, we employ Morera's theorem. Thus, let $\delta$ be the positively oriented circumference of a triangle $\Delta \subset U \backslash\{0\}$. If $\delta \subset \mathbf{D}_{+}$or $\delta \subset \mathbf{D}_{-}$, then $\int_{\delta} \Psi(z) d z=0$ because $\Psi$ is analytic in $\mathbf{D}_{+} \cap U$ and $\mathbf{D}_{-} \cap U$. If $\delta$ intersects $\mathbf{T}$, we can write $\delta=\delta_{+} \cup \delta_{-}$, where

$$
\delta_{+}=\left(\delta \cap \mathbf{D}_{+}\right) \cup \gamma, \quad \delta_{-}=\left(\delta \cap \mathbf{D}_{-}\right) \cup(-\gamma)
$$

$\gamma$ is the union of positively oriented subarcs of $\mathbf{T}$ and $-\gamma$ is $\gamma$ with the opposite orientation. Clearly,

$$
\int_{\delta_{+}} \Psi(z) d z=\int_{\delta_{+}} G_{+}(z) d z
$$


The matrix function $G_{+} \in\left[H_{+}^{2}(\mathbf{T})\right]_{n \times n} \subset\left[H_{+}^{1}(\mathbf{T})\right]_{n \times n}$ can be approximated by analytic polynomials $P_{m}^{+}$in the $H_{+}^{1}(\mathbf{T})$ norm as closely as desired, and because

$$
\int_{\delta_{+}} P_{m}^{+}(z) d z=0
$$

it follows that (3.2) also equals zero. Analogously,

$$
\int_{\delta_{-}} \Psi(z) d z=\int_{\delta_{-}} M^{-1}(z) G_{-}^{-1}(z) G(z) d z
$$

and $G_{-}^{-1} \in\left[H_{-}^{2}(\mathbf{T})\right]_{n \times n} \subset\left[H_{-}^{1}(\mathbf{T})\right]_{n \times n}$ can be approximated in $\left[H_{-}^{1}(\mathbf{T})\right]_{n \times n}$ by matrix functions of the form

$$
P_{m}^{-}(z)=p_{0}+p_{1} z^{-1}+\cdots+p_{m} z^{-m}
$$

as closely as wanted. As $M^{-1} P_{m}^{-} G$ is analytic in $U \backslash\{0\}$, we get

$$
\int_{\delta_{-}} M^{-1}(z) P_{m}^{-1}(z) G(z) d z=0
$$

which implies that (3.3) is also zero.

In summary, $\int_{\delta} \Psi(z) d z=0$ for every triangle curve $\delta=\partial \Delta$ with $\Delta \subset U \backslash\{0\}$. Hence, by Morera's theorem, $\Psi$ is analytic in $U \backslash\{0\}$. $\square$

Every real number $\alpha>0$ can be uniquely written in the form $\alpha=[\alpha]+\{\alpha\}$ where $[\alpha]$ is an integer and $\{\alpha\} \in[0,1)$. We denote by $C^{\alpha}$ the $[\alpha]$ times continuously differentiable function on $\mathbf{T}$ whose $[\alpha]$ th derivative satisfies a Hölder condition with the exponent $\{\alpha\}$.

Proposition 3.6. Let $G \in L_{n \times n}^{\infty}(\mathbf{T})$, let $\Gamma \subset \mathbf{T}$ be an open arc, and suppose $G$ is $C^{\alpha}, \alpha>0$, on $\Gamma$. If $T(G)$ is Fredholm, then $G$ possesses an $L^{2}$ factorization $G=G_{-} M G_{+}$such that $G_{ \pm}$and $G_{ \pm}^{-1}$ are also $C^{\alpha}$ on $\Gamma$.

Proof. The result is well known in the case where $\Gamma=\mathbf{T}$. So assume that $\Gamma$ is a proper subarc of $\mathbf{T}$, that is, $\Gamma=\left\{e^{i \theta}: \eta<\theta<\beta\right\}$ with 
$\beta-\eta<2 \pi$. Let $\gamma \subset \Gamma$ be any arc of the form $\gamma=\left\{e^{i \theta}: \eta_{0}<\theta<\beta_{0}\right\}$ with $\eta<\eta_{0}<\beta_{0}<\beta$. We must show that $G$ has an $L^{2}$ factorization $G=G_{-} M G_{+}$such that $G_{ \pm}, G_{ \pm}^{-1}$ are $C^{\alpha}$ on $\gamma$.

Since $T(G)$ is Fredholm, the matrix function $G$ is invertible in $L_{n \times n}^{\infty}(\mathbf{T})$. Hence, we can find an $n \times n$ matrix function $H$ whose entries are $C^{\alpha}$ on all of $\mathbf{T}$ such that $H|\gamma=G| \gamma$ and $\operatorname{det} H$ has no zeros on T. As $T(H)$ is Fredholm and as the proposition is true for $\Gamma=\mathbf{T}$, there is an $L^{2}$ factorization $H=H_{-} N H_{+}$with $H_{ \pm}, H_{ \pm}^{-1}$ in $C^{\alpha}$ on $\mathbf{T}$. Theorem 3.4 gives us an $L^{2}$ factorization $G=G_{-} M G_{+}$with $G_{ \pm}, G_{ \pm}^{-1}$ in $H_{ \pm}^{2}(\mathbf{T})$. Because $H=G$ on $\gamma$, it follows that

$$
M G_{+} H_{+}^{-1}=G_{-}^{-1} H_{-} N \quad \text { on } \gamma .
$$

Put

$$
\Psi(z):= \begin{cases}M(z) G_{+}(z) H_{+}^{-1}(z) & \text { for } z \in \mathbf{D}_{+} \backslash\{0\}, \\ G_{-}^{-1}(z) H_{-}(z) N(z) & \text { for } z \in \mathbf{D}_{-} \backslash\{\infty\},\end{cases}
$$

and let $\Psi$ be given by (3.4) on $\gamma$.

The matrix functions $G_{+}, G_{-}^{-1}, H_{+}^{-1}, H_{-}$can be approximated in $L^{2}$ by matrix functions of the form

$$
\begin{aligned}
& P_{m}^{+}(z)=p_{0}^{+}+p_{1}^{+} z+\cdots+p_{m}^{+} z^{m}, \\
& P_{m}^{-}(z)=p_{0}^{-}+p_{1}^{-} z^{-1}+\cdots+p_{m}^{-} z^{-m} \\
& Q_{m}^{+}(z)=q_{0}^{+}+q_{1}^{+} z+\cdots+q_{m}^{+} z^{m}, \\
& Q_{m}^{-}(z)=q_{0}^{-}+q_{1}^{-} z^{-1}+\cdots+q_{m}^{-} z^{-m}
\end{aligned}
$$

respectively, as closely as desired. Since $M P_{m}^{+} Q_{m}^{+}$and $P_{m}^{-} Q_{m}^{-} N$ are analytic in $\mathbf{D}_{+} \backslash\{0\}$ and $\mathbf{D}_{-} \backslash\{\infty\}$ and converge in $L^{1}$ to $M G_{+} H_{+}^{-1}$ and $G_{-}^{-1} H_{-} N$, respectively, we can employ Morera's theorem as in the proof of Proposition 3.5 to conclude that $\Psi$ is analytic in $\mathbf{C} \backslash\{0\}$.

Because $G_{+}=M^{-1} \Psi H_{+}$on $\gamma$, we see that $G_{+}$is $C^{\alpha}$ on $\gamma$. Since $G_{+}$ cannot have zeros on $\gamma$ (Theorem 3.2), it follows that $G_{+}^{-1}$ is also $C^{\alpha}$ on $\gamma$. Finally, since $G_{-}=G G_{+}^{-1} M^{-1}$, we arrive at the conclusion that $G_{-}^{ \pm}$are $C^{\alpha}$ on $\gamma$.

The following proposition is the Toeplitz analogue of the first part of Theorem 1.3. Recall that $\tilde{G}(t)=G(1 / t)$ for $t \in \mathbf{T}$. 
Proposition 3.7. If $G \in L_{n \times n}^{\infty}(\mathbf{T})$ has at most a finite number $m$ of harmless discontinuities and at most a finite number $d$ of severe discontinuities on $\mathbf{T}$ and if $T(G)$ and $T(\tilde{G})$ are Fredholm, then

$$
|\operatorname{Ind} T(G)+\operatorname{Ind} T(\tilde{G})| \leq d(n-1) .
$$

Proof. By Theorem 3.3, there is nothing to prove for $d=0$. Let us now assume that $G$ has exactly one discontinuity on $\mathbf{T}$, namely a severe discontinuity at $t_{0} \in \mathbf{T}$. We can approximate $G$ in $L_{n \times n}^{\infty}(\mathbf{T})$ as closely as desired by matrix functions which are $C^{1}$ on $\mathbf{T} \backslash\left\{t_{0}\right\}$. Since both the Fredholmness and the index are stable under uniform approximation, we may a priori assume that $G$ is $C^{1}$ on $\mathbf{T} \backslash\left\{t_{0}\right\}$. Applying Theorem 3.4 and Proposition 3.6 to $G$ and $\tilde{G}$, we get factorizations

$$
G=G_{-} M G_{+}=H_{+} N H_{-}
$$

where $G_{+}^{ \pm 1}, H_{+}^{ \pm 1} \in\left[H_{+}^{2}(\mathbf{T})\right]_{n \times n}, G_{-}^{ \pm 1}, H_{-}^{ \pm 1} \in\left[H_{-}^{2}(\mathbf{T})\right]_{n \times n}$,

$$
\begin{array}{rlrl}
M(t) & =\operatorname{diag}\left(t^{\varrho 1}, \ldots, t^{\varrho n}\right), & \varrho:=\varrho_{1}+\cdots+\varrho_{n}=-\operatorname{Ind} T(G), \\
N(t) & =\operatorname{diag}\left(t^{\lambda_{1}}, \ldots, t^{\lambda_{n}}\right), & & \lambda:=\lambda_{1}+\cdots+\lambda_{n}=\operatorname{Ind} T(\tilde{G}),
\end{array}
$$

and $G_{+}^{ \pm 1}, H_{+}^{ \pm 1}, G_{-}^{ \pm 1}, H_{-}^{ \pm 1}$ are $C^{1}$ on $\mathbf{T} \backslash\left\{t_{0}\right\}$. Taking the determinant of (3.5), we obtain

$$
\operatorname{det} G_{-}(t) t^{\varrho} \operatorname{det} G_{+}(t)=\operatorname{det} H_{+}(t) t^{\lambda} \operatorname{det} H_{-}(t),
$$

whence

$$
t^{\varrho-\lambda} \varphi_{+}(t)=\varphi_{-}(t), \quad \varphi_{+}:=\frac{\operatorname{det} G_{+}}{\operatorname{det} H_{+}}, \quad \varphi_{-}:=\frac{\operatorname{det} H_{-}}{\operatorname{det} G_{-}} .
$$

Obviously, $\operatorname{det} G_{+}^{ \pm 1} \in H_{+}^{2 / n}(\mathbf{T})$ and $\operatorname{det} H_{-}^{ \pm 1} \in H_{-}^{2 / n}(\mathbf{T})$. This shows that $\varphi_{ \pm} \in H_{ \pm}^{1 / n}(\mathbf{T})$. Since $\varphi_{ \pm}(z) \neq 0$ for $z \in \mathbf{D}_{ \pm}$and $\varphi_{ \pm}$are nonzero and continuous on $\mathbf{T} \backslash\left\{t_{0}\right\}$, there are functions $\psi_{ \pm}$such that

$$
\left(\psi_{ \pm}(z)\right)^{n}=\varphi_{ \pm}(z) \quad \text { for } z \in \mathbf{D}_{ \pm} \cup\left(\mathbf{T} \backslash\left\{t_{0}\right\}\right)
$$


and $\psi_{ \pm}$are analytic in $\mathbf{D}_{ \pm}$and continuous on $\mathbf{T} \backslash\left\{t_{0}\right\}$. Because $\varphi_{ \pm} \in H_{ \pm}^{1 / n}(\mathbf{T})$ and hence

$$
\sup _{0<r^{ \pm 1}<1} \int_{0}^{2 \pi}\left|\psi_{ \pm}\left(r e^{i \theta}\right)\right| d \theta=\sup _{0<r^{ \pm 1}<1} \int_{0}^{2 \pi}\left|\varphi_{ \pm}\left(r e^{i \theta}\right)\right|^{1 / n} d \theta<\infty
$$

it results that $\psi_{ \pm} \in H_{ \pm}^{1}(\mathbf{T})$. Analogously, one can show that $\psi_{ \pm}^{-1}$ are in $H^{1}(\mathbf{T})$.

Our aim is to prove that $|\varrho-\lambda| \leq n-1$. Contrary to what we want, let us assume that $|\varrho-\lambda| \geq n$. For the sake of definiteness, suppose $\varrho-\lambda=n+\alpha$ with an integer $\alpha \geq 0$ (the case $\varrho-\lambda \leq-n$ can be reduced to the case considered here by passage to adjoint operators). Let $t^{\alpha / n}$ be any branch of the function which is continuous on $\mathbf{T} \backslash\left\{t_{0}\right\}$. From (3.6) and (3.7) we obtain

$$
\left(t t^{\alpha / n} \psi_{+}(t)\right)^{n}=\left(\psi_{-}(t)\right)^{n} \quad \text { for } t \in \mathbf{T} \backslash\left\{t_{0}\right\} .
$$

Taking into account that $t t^{n / \alpha} \psi_{+}(t)$ and $\psi_{-}(t)$ are continuous on $\mathbf{T} \backslash\left\{t_{0}\right\}$, we therefore get

$$
t t^{\alpha / n} \psi_{+}(t)=c \psi_{-}(t) \quad \text { for } t \in \mathbf{T} \backslash\left\{t_{0}\right\},
$$

where $c \in \mathbf{C}$ satisfies $c^{n}=1$. We now employ an old trick, namely, we write

$$
t^{\alpha / n}=\left(t-t_{0}\right)^{\alpha / n}\left(1-t_{0} / t\right)^{-\alpha / n},
$$

which is valid with appropriately chosen branches of $\left(t-t_{0}\right)^{\alpha / n}$ and $\left(1-t_{0} / t\right)^{-a / n}$. It follows that

$$
t\left(t-t_{0}\right)^{\alpha / n} \psi_{+}(t)=c\left(1-t_{0} / t\right)^{\alpha / n} \psi_{-}(t) \quad \text { for } t \in \mathbf{T} \backslash\left\{t_{0}\right\} .
$$

Since $\psi_{ \pm} \in H_{ \pm}^{1}(\mathbf{T})$ and

$$
\left(t-t_{0}\right)^{\alpha / n} \in H_{ \pm}^{\infty}(\mathbf{T}), \quad\left(t-t_{0} / t\right)^{\alpha / n} \in H_{-}^{\infty}(\mathbf{T})
$$

we see that the lefthand side of $(3.8)$ belongs to $H_{+}^{1}(\mathbf{T})$, while the righthand side of $(3.8)$ is a function in $H_{-}^{1}(\mathbf{T})$. As $H_{+}^{1}(\mathbf{T}) \cap H_{-}^{1}(\mathbf{T})$ is the set of all constant functions, the function

$$
t\left(t-t_{0}\right)^{\alpha / n} \psi_{+}(t) \in H_{+}^{1}(\mathbf{T})
$$


must be constant. This function vanishes at the origin, whence

$$
t\left(t-t_{0}\right)^{\alpha / n} \psi_{+}(t)=0 \quad \text { for } t \in \mathbf{T} \backslash\left\{t_{0}\right\},
$$

which is impossible because $\psi_{+}^{-1} \in H_{+}^{1}(\mathbf{T})$. This contradiction completes the proof in the case where $G$ has exactly one severe discontinuity and no other discontinuities.

If $G$ has exactly $d$ severe discontinuities and exactly $m$ harmless discontinuities $\mathbf{T}$, we can write

$$
G=G_{1} \cdots G_{d} G_{d+1} \cdots G_{d+m}
$$

where each $G_{j}$ has exactly one discontinuity on $\mathbf{T}$, which is a severe discontinuity for $1 \leq j \leq d$ and a harmless discontinuity for $d+1 \leq$ $j \leq d+m$. A simple application of Theorems 3.2 and 3.3 shows that all $T\left(G_{j}\right)$ and $T\left(\tilde{G}_{j}\right)$ are Fredholm together with $T(G)$ and $T(\tilde{G})$. Also by Theorem 3.2, the operators

$$
T(G)-\prod_{j=1}^{d+m} T\left(G_{j}\right) \quad \text { and } \quad T(\tilde{G})-\prod_{j=1}^{d+m} T\left(\tilde{G}_{j}\right)
$$

are compact. Consequently, from what was already proved, we get

$$
\begin{aligned}
|\operatorname{Ind} T(G)+\operatorname{Ind} T(\tilde{G})|= & \mid \sum_{j=1}^{d} \operatorname{Ind} T\left(G_{j}\right)+\sum_{j=1}^{d} \operatorname{Ind} T\left(\tilde{G}_{j}\right) \\
& +\sum_{j=d+1}^{d+m} \operatorname{Ind} T\left(G_{j}\right)+\sum_{j=d+1}^{d+m} \operatorname{Ind} T\left(\tilde{G}_{j}\right) \mid \\
= & \left|\sum_{j=1}^{d} \operatorname{Ind} T\left(G_{j}\right)+\sum_{j=1}^{d} \operatorname{Ind} T\left(\tilde{G}_{j}\right)\right| \\
\leq & \sum_{j=1}^{d}\left|\operatorname{Ind} T\left(G_{j}\right)+\operatorname{Ind} T\left(\tilde{G}_{j}\right)\right| \\
\leq & d(n-1) .
\end{aligned}
$$

Combining Lemma 3.1 and Proposition 3.7 we obtain the first assertion of Theorem 1.3. 
4. Bounded symbols with prescribed total indices. We now proceed to the proof of the second part of Theorem 1.3.

A matrix function $F \in L_{n \times n}^{\infty}(\mathbf{R})$ is said to be uniformly positive definite if there is an $\varepsilon>0$ such that $(F(x) \zeta, \zeta) \geq \varepsilon\|\zeta\|^{2}$ for all $\zeta \in \mathbf{C}^{n}$ and almost all $x \in \mathbf{R}$.

Theorem 4.1. A matrix function $F \in L_{n \times n}^{\infty}(\mathbf{R})$ is uniformly positive definite if and only if it can be represented in the form $\mathrm{F}=\mathrm{H}_{+} \mathrm{H}_{+}^{*}$ with $H_{+} \in G\left[H_{+}^{\infty}(\mathbf{R})\right]_{n \times n}$.

For a proof of this well-known fact, see [5] or [11].

Recall that $C(\overline{\mathbf{R}})$ is the set of all continuous functions $g$ on $\mathbf{R}$ which have finite limits $g( \pm \infty)$ at $\pm \infty$. The following well-known theorem provides us with a Fredholm criterion and an index formula for WienerHopf operators with symbols in $[C(\overline{\mathbf{R}})]_{n \times n}$.

Theorem 4.2. Let $F \in[C(\overline{\mathbf{R}})]_{n \times n}$. For $W(F)$ to be Fredholm it is necessary and sufficient that $\operatorname{det} F(x) \neq 0$ for all $x \in \mathbf{R} \cup\{ \pm \infty\}$ and that none of the eigenvalues $\xi_{1}, \ldots, \xi_{n}$ of $F^{-1}(-\infty) F(+\infty)$ is located on $(-\infty, 0]$. If $W(F)$ is Fredholm, then

$$
\text { Ind } W(F)=-\frac{1}{2 \pi}\{\arg \operatorname{det} F\}_{-\infty}^{\infty}+\frac{1}{2 \pi} \sum_{j=1}^{n} \arg \xi_{j},
$$

where $\{\arg \operatorname{det} F\}_{-\infty}^{\infty}$ stands for the increment of any continuous branch of the argument $\arg \operatorname{det} F(x)$ of $\operatorname{det} F(x)$ as $x$ moves from $-\infty$ to $+\infty$ and where $\arg \xi_{j}$ is the argument of $\xi_{j}$ taken in $(-\pi, \pi)$.

Proofs can be found in $[4,5,11]$.

We know from Lemma 3.1 and Theorem 3.3 that if $A \in[C(\overline{\mathbf{R}})]_{n \times n}$ and $W(A)$ as well as $W(\tilde{A})$ are Fredholm, then

$$
\text { Ind } W(A)+\operatorname{Ind} W(\tilde{A})=0 \text {. }
$$

It should be mentioned that this can also be easily deduced from Theorem 4.2. Indeed, let $\xi_{j}$ be the eigenvalues of $A^{-1}(-\infty) A(+\infty)$. Since

$$
\tilde{A}^{-1}(-\infty) \tilde{A}(+\infty)=A^{-1}(+\infty) A(-\infty)
$$


the eigenvalues $\tilde{\xi}_{j}$ of $\tilde{A}^{-1}(-\infty) \tilde{A}(+\infty)$ are $\tilde{\xi}_{j}=1 / \xi_{j}$, whence $\arg \xi_{j}=$ $-\arg \tilde{\xi}_{j}$. From Theorem 4.2 we therefore obtain

$$
\begin{aligned}
& \text { Ind } W(A)=-\frac{1}{2 \pi}\{\arg \operatorname{det} A\}_{-\infty}^{\infty}+\frac{1}{2 \pi} \sum_{j=1}^{n} \arg \xi_{j}, \\
& \text { Ind } W(\tilde{A})=\frac{1}{2 \pi}\{\arg \operatorname{det} A\}_{-\infty}^{\infty}-\frac{1}{2 \pi} \sum_{j=1}^{n} \arg \xi_{j},
\end{aligned}
$$

and addition of these two equalities gives (4.2).

The third main ingredient of our proof is the following beautiful result by Ballantine $[\mathbf{1}, \mathbf{2}]$, also see $[\mathbf{8}$, p. 295].

Theorem 4.3 (Ballantine). A matrix $M \in \mathbf{C}^{n \times n}$ is the product of four positive definite matrices in $\mathbf{C}^{n \times n}$ if and only if

$$
\operatorname{det} M>0 \quad \text { and } \quad M \notin \bigcup_{\lambda<0}\left\{\lambda I_{n}\right\},
$$

where $I_{n}$ is the $n \times n$ identity matrix.

The following result is the key to the proof of the second assertion of Theorem 1.3.

Proposition 4.4. Let $n \geq 2$ be a natural number, and let $\nu \in$ $\{-(n-1), \ldots, n-1\}$. Then there exists an $A \in L_{n \times n}^{\infty}(\mathbf{R})$ which is continuous on $\mathbf{R}$ and extends to an analytic function in $\mathbf{C} \backslash\{-i, i\}$ such that $W(A)$ is invertible and $W(\tilde{A})$ is Fredholm of index $\nu$.

Proof. Without loss of generality, assume $\nu \in\{1, \ldots, n-1\}$; the case $\nu=0$ is trivial and the case $\nu \in\{-1, \ldots,-(n-1)\}$ can be disposed of by passage to adjoint operators. Let $k:=n-\nu$, and let $M$ be the $n \times n$ diagonal matrix

$$
M:=\operatorname{diag}\left(e^{-2 \pi i k / n}, \ldots, e^{-2 \pi i k / n}\right) .
$$

By Theorem 4.3, there are four positive definite matrices $B( \pm \infty)$, $C( \pm \infty)$ such that

$$
M=C^{-1}(+\infty) B^{-1}(+\infty) B(-\infty) C(-\infty) .
$$


Define $B, C \in[C(\overline{\mathbf{R}})]_{n \times n}$ by

$$
\begin{aligned}
& B(x)=(1-u(x)) B(-\infty)+u(x) B(+\infty), \\
& C(x)=(1-u(x)) C(-\infty)+u(x) C(+\infty),
\end{aligned}
$$

where $u(x):=(\pi / 2+\arctan x) / \pi$. Clearly, $B$ and $C$ extend to analytic functions in $\mathbf{C} \backslash\{-i, i\}$. Since $0 \leq u \leq 1$ on $\mathbf{R}$, we also see that there is an $\varepsilon>0$ such that

$$
(B(x) \zeta, \zeta) \geq \varepsilon\|\zeta\|^{2} \quad \text { and } \quad(C(x), \zeta, \zeta) \geq \varepsilon\|\zeta\|^{2}
$$

for all $x \in \mathbf{R}$ and all $\zeta \in \mathbf{C}^{n}$. From Theorem 4.1 we therefore deduce the existence of $B_{+}, C_{+} \in G\left[H_{+}^{\infty}(\mathbf{R})\right]_{n \times n}$ such that $B=B_{+} B_{+}^{*}$ and $C=C_{+} C_{+}^{*}$. Taking into account Lemma 3.1 and Proposition 3.5, it is easily seen that $B_{+}, C_{+}$extend to analytic functions in $\mathbf{C} \backslash\{-i, i\}$.

For a real number $\delta \in(0,1 / 2)$, define $\Lambda \in[C(\overline{\mathbf{R}})]_{n \times n}$ by

$$
\Lambda(x):=\operatorname{diag}\left(\left(\frac{x-i}{x+i}\right)^{\delta}, \ldots,\left(\frac{x-i}{x+i}\right)^{\delta}\right), \quad x \in \mathbf{R} .
$$

The branch of $((x-i) /(x+i))^{\delta}$ is chosen so that

$$
\Lambda(-\infty)=I_{n} \quad \text { and } \quad \Lambda(+\infty)=e^{2 \pi i \delta} I_{n} .
$$

The operator $W\left(((x-i) /(x+i))^{\delta}\right)$ is known to be invertible, and hence $W(\Lambda)$ is also invertible.

Put $A:=B_{+}^{*} \Lambda C_{+}$. Clearly, $W(A)=W\left(B_{+}^{*}\right) W(\Lambda) W\left(C_{+}\right)$is invertible, $A$ is continuous on $\mathbf{R}$, and $A$ extends to an analytic function in $\mathbf{C} \backslash\{-i, i\}$. Since

$$
\begin{aligned}
W\left(\tilde{B}_{+}\right) W(\tilde{A}) W\left(\tilde{C}_{+}^{*}\right) & =W\left(\tilde{B}_{+}\right) W\left(\tilde{B}_{+}^{*} \tilde{\Lambda} \tilde{C}_{+}\right) W\left(\tilde{C}_{+}^{*}\right) \\
& =W\left(\tilde{B}_{+} \tilde{B}_{+}^{*} \tilde{\Lambda} \tilde{C}_{+} \tilde{C}_{+}^{*}\right) \\
& =W(\tilde{B} \tilde{\Lambda} \tilde{C})
\end{aligned}
$$

$W(\tilde{A})$ is Fredholm if and only if $W(\tilde{B} \tilde{\Lambda} \tilde{C})$ is Fredholm, in which case both operators have the same index. 
As $\tilde{B} \tilde{\Lambda} \tilde{C} \in[C(\overline{\mathbf{R}})]_{n \times n}$, the Fredholm properties of $W(\tilde{B} \tilde{\Lambda} \tilde{C})$ can be studied with the help of Theorem 4.2. It is clear that $\operatorname{det}(\tilde{B} \tilde{\Lambda} \tilde{C})$ does not vanish on $\mathbf{R} \cup\{ \pm \infty\}$. Moreover,

$$
\begin{aligned}
-\frac{1}{2 \pi}\{\arg \operatorname{det}(\tilde{B} \tilde{\Lambda} \tilde{C})\}_{-\infty}^{\infty}= & \frac{1}{2 \pi}\{\arg \operatorname{det}(B \Lambda C)\}_{-\infty}^{\infty} \\
= & \frac{1}{2 \pi}\{\arg \operatorname{det} B\}_{-\infty}^{\infty}+\frac{1}{2 \pi}\{\arg \operatorname{det} \Lambda\}_{-\infty}^{\infty} \\
& +\frac{1}{2 \pi}\{\arg \operatorname{det} C\}_{-\infty}^{\infty} \\
= & 0+\frac{1}{2 \pi} n \cdot 2 \pi \delta+0=n \delta .
\end{aligned}
$$

We have

$$
\begin{aligned}
(\tilde{B} \tilde{\Lambda} \tilde{C})^{-1} & (-\infty)(\tilde{B} \tilde{\Lambda} \tilde{C})(+\infty) \\
& =(B \Lambda C)^{-1}(+\infty)(B \Lambda C)(-\infty) \\
& =C^{-1}(+\infty) \Lambda^{-1}(+\infty) B^{-1}(+\infty) B(-\infty) \Lambda(-\infty) C(-\infty) \\
& =C^{-1}(+\infty) e^{-2 \pi i \delta} I_{n} B^{-1}(+\infty) B(-\infty) I_{n} C(-\infty) \\
& =e^{-2 \pi i \delta} M=\operatorname{diag}\left(e^{2 \pi i(-k / n-\delta)}, \ldots, e^{2 \pi i(-k / n-\delta)}\right) .
\end{aligned}
$$

The eigenvalues of this matrix are

$$
\xi_{1}=\cdots=\xi_{n}=e^{2 \pi i(-k / n-\delta)} .
$$

Now choose $\delta \in(0,1 / 2)$ so that $-1<-k / n-\delta<-1 / 2$ (recall that $k \in\{1, \ldots, n-1\})$. Then $0<2 \pi(-k / n-\delta)+2 \pi<\pi$, and hence the argument $\arg \xi_{j}$ lying in $(-\pi, \pi)$ is $2 \pi(-k / n-\delta)+2 \pi$. This together with (4.3) and Theorem 4.2 yields that $W(\tilde{B} \tilde{\Lambda} \tilde{C})$ is Fredholm and that

$$
\text { Ind } W(\tilde{B} \tilde{\Lambda} \tilde{C})=n \delta+n(-k / n-\delta)+n=n-k=\nu . \quad \square
$$

We are now in a position to complete the proof of Theorem 1.3. Combining Proposition 4.4 and Lemma 3.1, we get matrix functions $G \in L_{n \times n}^{\infty}(\mathbf{T})$ with at most a discontinuity at $t_{0}=1$ such that $T(G)$ is invertible and thus Fredholm of index zero, whereas $T(\tilde{G})$ is Fredholm with any prescribed index in $\{-(n-1), \ldots, n-1\}$. On replacing $G(t)$ 
by $G\left(t / t_{0}\right)$ with $t_{0} \in \mathbf{T}$, which amounts to replacing the matrix (3.1) by

$$
\begin{aligned}
\left(\begin{array}{cccl}
G_{0} & t_{0} G-1 & t_{0}^{2} G_{-2} & \cdots \\
t_{0}^{-1} G_{1} & G_{0} & t_{0} G_{-1} & \ldots \\
t_{0}^{-2} G_{2} & t_{0}^{-1} G_{1} & G_{0} & \cdots \\
\cdots & \cdots & \cdots & \ldots
\end{array}\right) \\
\\
=D_{t_{0}}^{-1}\left(\begin{array}{cccc}
G_{0} & G_{-1} & G_{-2} & \ldots \\
G_{1} & G_{0} & G_{-1} & \ldots \\
G_{2} & G_{1} & G_{0} & \ldots \\
\ldots & \ldots & \ldots & \ldots
\end{array}\right) D_{t_{0}}
\end{aligned}
$$

with $D_{t_{0}}:=\operatorname{diag}\left(1, t_{0}, t_{0}^{2}, \cdots\right)$, we can achieve that $G$ has its discontinuity at an arbitrarily given point $t_{0} \in \mathbf{T}$. Using Lemma 3.1 to go back to the real line, we obtain $A \in L_{n \times n}^{\infty}(\mathbf{R})$ with at most a discontinuity at a given point $x_{0} \in \mathbf{R} \cup\{\infty\}$ such that $W(A)$ is Fredholm of index zero and $W(\tilde{A})$ is Fredholm of prescribed index $\nu \in\{-(n-1), \ldots, n-1\}$. Considering products $A:=A_{1} \cdots A_{d}$ in which each $A_{j}$ has at most one discontinuity and $A_{j}, A_{k}$ have no common discontinuity for $j \neq k$ and taking into account Theorem 3.2, we arrive at $A \in L_{n \times n}^{\infty}(\mathbf{R})$ with at most $d$ discontinuities on $\mathbf{R} \cup\{\infty\}$ for which $W(A)$ is Fredholm of index zero and $W(\tilde{A})$ is Fredholm of prescribed index $\nu \in\{-d(n-1), \ldots, d(n-1)\}$. Replacing $A(x)$ by

$$
A(x) \operatorname{diag}\left(\left(\frac{x+i}{x-i}\right)^{\kappa}, 1, \ldots, 1\right)
$$

we get the desired $A$ 's with

$$
\text { Ind } W(A)=\kappa
$$

and

$$
\text { Ind } W(\tilde{A}) \in\{-\kappa-d(n-1), \ldots,-\kappa+d(n-1)\} \text {. }
$$

5. Semi-almost periodic symbols. In this section we prove Theorem 1.6. For this purpose we have to recall some well-known results on Wiener-Hopf operators with almost and with semi-almost periodic symbols. 
Let $A P W$ denote the collection of all almost periodic functions $f$ with absolutely convergent Fourier series:

$$
f(x)=\sum_{j} f_{j} e^{i \lambda_{j} x}, \quad \lambda_{j} \in \mathbf{R}, \quad \sum_{j}\left|f_{j}\right|<\infty .
$$

For $F \in A P W_{n \times n}$, the matrix coefficient corresponding to $\lambda_{j}=0$ is referred to as the mean value of $F$ and is denoted by $\mathbf{M}(F)$; in other terms,

$$
\mathbf{M}(F)=\lim _{T \rightarrow \infty} \frac{1}{2 T} \int_{-T}^{T} F(x) d x .
$$

A matrix function $F \in A P W_{n \times n}$ is said to possess a right canonical $A P W$ factorization if it can be represented in the form $F=F_{-} F_{+}$ where

$$
F_{ \pm} F_{ \pm}^{-1} \in A P W_{n \times n} \cap\left[H_{ \pm}^{\infty}(\mathbf{R})\right]_{n \times n}
$$

and is said to have a left canonical $A P W$ factorization if it can be written in the form $F=H_{+} H_{-}$where

$$
H_{ \pm} H_{ \pm}^{-1} \in A P W_{n \times n} \cap\left[H_{ \pm}^{\infty}(\mathbf{R})\right]_{n \times n} .
$$

It is well known that if a right canonical $A P W$ factorization of $F$ exists, then the factors $F_{-}, F_{+}$are uniquely determined up to the transformation $F_{-} \mapsto F_{-} C, F_{+} \mapsto C^{-1} F_{+}$with an arbitrary invertible matrix $C \in \mathbf{C}^{n \times n}$. Thus, if $F$ has a right canonical $A P W$ factorization $F=F_{-} F_{+}$, then the so-called right geometric mean $\mathbf{d}_{r}(F):=\mathbf{M}\left(F_{-}\right) \mathbf{M}\left(F_{+}\right)$is well defined. Analogously, in case $F$ has a left canonical $A P W$ factorization $F=H_{+} H_{-}$, the left geometric mean is the well-defined matrix $\mathbf{d}_{l}(F):=\mathbf{M}\left(H_{+}\right) \mathbf{M}\left(H_{-}\right)$.

Theorem 5.1. For $F \in A P W_{n \times n}$ the following are equivalent:

(i) $W(F)$ is Fredholm;

(ii) $W(F)$ is invertible;

(iii) $F$ has a right canonical $A P W$ factorization.

We denote by $S A P W_{n \times n}$ the set of all matrix functions $F$ of the form

$$
F(x)=(1-u(x)) F_{l}(x)+u(x) F_{r}(x)+F_{0}(x)
$$


where $u \in C(\overline{\mathbf{R}})$ is a fixed function such that $0 \leq u \leq 1, u(-\infty)=0$, $u(+\infty)=1$, where $F_{l}, F_{r} \in A P W_{n \times n}$, and where $F_{0} \in[C(\dot{\mathbf{R}})]_{n \times n}$ is a matrix function for which $F_{0}(\infty)$ is the zero matrix. The matrix functions $F_{l}$ and $F_{r}$ are called the almost periodic representatives of $F$ at $-\infty$ and $+\infty$, respectively. One can show that $S A P W_{n \times n}$ is a dense subset of $S A P_{n \times n}$, see [12].

Theorem 5.2. Let $F \in S A P W_{n \times n}$. The operator $W(F)$ is Fredholm if and only if the following three conditions are satisfied:

(a) $\operatorname{det} F(x) \neq 0$ for all $x \in \mathbf{R}$;

(b) $F_{l}$ and $F_{r}$ have right canonical $A P W$ factorizations;

(c) none of the eigenvalues $\xi_{1}, \ldots, \xi_{n}$ of $\left(\mathbf{d}_{r}\left(F_{l}\right)\right)^{-1} \mathbf{d}_{r}\left(F_{r}\right)$ lies on $(-\infty, 0]$.

In that case the index of $W(F)$ is given by formula (4.1).

The scalar versions of Theorems 5.1 and 5.2 are due to Coburn, Douglas, Gohberg, Feldman and Sarason. In the matrix case, these theorems were established in $[\mathbf{9}]$ and $[\mathbf{1 0}]$.

We remark that Theorem 5.2 immediately gives the $S A P$ version of the first assertion of Theorem 1.3, that is, the inequality

$$
|\operatorname{Ind} W(A)+\operatorname{Ind} W(\tilde{A})| \leq n-1
$$

Indeed, since $S A P W_{n \times n}$ is dense in $S A P_{n \times n}$, it suffices to prove the inequality for $A$ in $S A P W_{n \times n}$. In that case we obtain from Theorem 5.2 that

$$
\begin{aligned}
& \text { Ind } W(A)=-\frac{1}{2 \pi}\{\arg \operatorname{det} A\}_{-\infty}^{\infty}+\frac{1}{2 \pi} \sum_{j=1}^{n} \arg \xi_{j}, \\
& \text { Ind } W(\tilde{A})=\frac{1}{2 \pi}\{\arg \operatorname{det} A\}_{-\infty}^{\infty}+\frac{1}{2 \pi} \sum_{j=1}^{n} \arg \tilde{\xi}_{j},
\end{aligned}
$$

where $\arg \xi_{j}$ and $\arg \tilde{\xi}_{j}$ are certain numbers in $(-\pi, \pi)$. Adding these two equalities we get

$$
|\operatorname{Ind} W(A)+\operatorname{Ind} W(\tilde{A})|<\frac{1}{2 \pi}(n \pi+n \pi)=n,
$$


which completes the proof.

If $F \in A P W_{n \times n}$ is uniformly positive definite, then $F$ has both a left and a right canonical $A P W$ factorization, the geometric means $\mathbf{d}_{l}(F)$ and $\mathbf{d}_{r}(F)$ are positive definite matrices and, obviously,

$$
\operatorname{det} \mathbf{d}_{l}(F)=\operatorname{det} \mathbf{d}_{r}(F)=\exp \left(\frac{1}{2 \pi} \int_{-\pi}^{\pi} \log \operatorname{det} F\left(e^{i \theta}\right) d \theta\right) .
$$

The following result shows that the equality of the determinants is the only general relation between the left and right geometric means of uniformly positive definite matrix functions in $S A P W_{n \times n}$.

Lemma 5.3. Given any two positive definite matrices $D_{l}$ and $D_{r}$ in $\mathbf{C}^{n \times n}$ for which $\operatorname{det} D_{l}=\operatorname{det} D_{r}$, there exists a $2 \pi$-periodic uniformly positive definite matrix function $F$ in $A P W_{n \times n}$ such that

$$
\mathbf{d}_{l}(F)=D_{l} \quad \text { and } \quad \mathbf{d}_{r}(F)=D_{r} .
$$

Proof. It is again more convenient to pass from the real line to the unit circle $\mathbf{T}$. Let $W$ denote the Wiener algebra of all functions $g$ on $\mathbf{T}$ with absolutely convergent Fourier series,

$$
g(t)=\sum_{n \in \mathbf{Z}} g_{n}\left(t^{n}\right), \quad t \in \mathbf{T} \quad \text { with } \quad \sum_{n \in \mathbf{Z}}\left|g_{n}\right|<\infty,
$$

and put $W^{ \pm}:=W \cap H_{ \pm}^{\infty}(\mathbf{T})$. A right, respectively left, canonical $W$ factorization of $G \in W_{n \times n}$ is a representation $G=G_{-} G_{+}$, respectively $G=K_{+} K_{-}$, with $G_{ \pm}, G_{ \pm}^{-1}$ in $W_{n \times n}^{ \pm}$, respectively with $K_{ \pm}, K_{ \pm}^{-1}$ in $W_{n \times n}^{ \pm}$. Given a right canonical $W$ factorization of $G=G_{-} G_{+}$, we define the right geometric mean $\mathbf{d}_{r}(G)$ as $G_{-}(\infty) G_{+}(0)$. Notice that the functions in $W^{ \pm}$extend to analytic functions in $\mathbf{D}_{ \pm}$. We also remark that the definition of $\mathbf{d}_{r}(G)$ is independent of the particular $W$ factorization $G=G_{-} G_{+}$. Analogously, if $G=K_{+} K_{-}$is a left canonical $W$ factorization, the left geometric mean is defined by $\mathbf{d}_{l}(G)=K_{+}(0) K_{-}(\infty)$.

We will construct a positive definite matrix function $G \in W_{n \times n}$ which has left and right canonical $W$ factorizations $G=K_{+} K_{-}=G_{-} G_{+}$such that

$$
\mathbf{d}_{l}(G)=K_{+}(0) K_{-}(\infty)=D_{l}, \quad \mathbf{d}_{r}(G)=G_{-}(\infty) G_{+}(0)=D_{r} .
$$


Letting $F(x):=G\left(e^{i x}\right)$, we get a $2 \pi$-periodic and uniformly positive definite matrix function $F \in A P W_{n \times n}$. Clearly,

$$
F(x)=K_{+}\left(e^{i x}\right) K_{-}\left(e^{i x}\right)=G_{-}\left(e^{i x}\right) G_{+}\left(e^{i x}\right)
$$

are canonical $A P W$ factorizations of $F$, and since

$$
\begin{aligned}
\mathbf{d}_{l}(F) & =\mathbf{M}\left(K_{+}\left(e^{i x}\right)\right) \mathbf{M}\left(K_{-}\left(e^{i x}\right)\right)=K_{+}(0) K_{-}(\infty), \\
\mathbf{d}_{r}(F) & =\mathbf{M}\left(G_{-}\left(e^{i x}\right)\right) \mathbf{M}\left(G_{+}\left(e^{i x}\right)\right)=G_{-}(\infty) G_{+}(0),
\end{aligned}
$$

we arrive at (5.1).

To construct $G$, let $U$ be a unitary matrix diagonalizing the marix $D_{l}^{-1 / 2} D_{r} D_{l}^{-1 / 2}$, that is,

$$
D_{l}^{-1 / 2} D_{r} D_{l}^{-1 / 2}=U^{*} \Lambda U
$$

Suppose we have a positive definite matrix function $R$ for which

$$
\mathbf{d}_{l}(R)=I, \quad \mathbf{d}_{r}(R)=\Lambda
$$

Then $G:=D_{l}^{1 / 2} U^{*} R U D_{l}^{1 / 2}$ is a positive definite matrix function satisfying (5.2). Indeed,

$$
\begin{aligned}
\mathbf{d}_{l}(G) & =D_{l}^{1 / 2} U^{*} \mathbf{d}_{l}(R) U D_{l}^{1 / 2}=D_{l}^{1 / 2} U^{*} U D_{l}^{1 / 2}=D_{l}, \\
\mathbf{d}_{r}(G) & =D_{l}^{1 / 2} U^{*} \mathbf{d}_{r}(R) U D_{l}^{1 / 2}=D_{l}^{1 / 2} U^{*} \Lambda U D_{l}^{1 / 2} \\
& =D_{l}^{1 / 2} D_{l}^{-1 / 2} D_{r} D_{l}^{-1 / 2} D_{l}^{1 / 2}=D_{r}
\end{aligned}
$$

In other words, it suffices to construct a positive definite matrix function $R$ having property (5.3).

Observe that $\Lambda=\operatorname{diag}\left(\lambda_{1}, \ldots, \lambda_{n}\right)$ with $\lambda_{j}>0$ and

$$
\lambda_{1} \cdots \lambda_{n}=\operatorname{det} \Lambda=\operatorname{det}\left(D_{l}^{-1 / 2} D_{r} D_{l}^{-1 / 2}\right)=1 .
$$

We will be looking for $R$ in the form

$$
R(z)=(I+z A)(I+z A)^{*}, \quad|z|=1,
$$


where $A$ is a matrix all eigenvalues of which belong to $\mathbf{D}_{+}$. Then, of course, (5.4) is a left canonical $W$ factorization of $R$ and $\mathbf{d}_{l}(R)=I$. Suppose that at the same time

$$
R(z)=\left(\Lambda^{1 / 2}+z B\right)^{*}\left(\Lambda^{1 / 2}+z B\right), \quad|z|=1,
$$

and $\Lambda^{-1 / 2} B$ has all its eigenvalues in $\mathbf{D}_{+}$. Then (5.5) delivers a right canonical $W$ factorization of $R$ with $\mathbf{d}_{r}(R)=\Lambda^{1 / 2} \Lambda^{1 / 2}=\Lambda$.

For (5.4) and (5.5) to hold simultaneously it is necessary and sufficient that

$$
I+A A^{*}=\Lambda+B^{*} B \text { and } A=\Lambda^{1 / 2} B,
$$

which in turn leads to the equation

$$
I+\Lambda^{1 / 2} B B^{*} \Lambda^{1 / 2}=\Lambda+B^{*} B .
$$

Let $B$ be the matrix of a weighted shift transformation,

$$
B=\left(\begin{array}{ccccc}
0 & b_{1} & 0 & \cdots & 0 \\
0 & 0 & b_{2} & \cdots & 0 \\
& & & \ddots & \\
0 & 0 & 0 & \cdots & b_{n-1} \\
b_{n} & 0 & 0 & \cdots & 0
\end{array}\right),
$$

with positive weights $b_{j}$ to be specified later. Then

$$
B B^{*}=\operatorname{diag}\left(b_{1}^{2}, \ldots, b_{n}^{2}\right), \quad B^{*} B=\operatorname{diag}\left(b_{n}^{2}, b_{1}^{2}, \ldots, b_{n-1}^{2}\right),
$$

so that (5.6) takes the form

$$
\begin{aligned}
1+\lambda_{j+1} b_{j+1}^{2} & =\lambda_{j+1}+b_{j}^{2}, \quad j=1, \ldots, n-1, \\
1+\lambda_{1} b_{1}^{2} & =\lambda_{1}+b_{n}^{2} .
\end{aligned}
$$

Choose a number $\varepsilon \in(0,1)$ smaller than all the products $\prod_{j=2}^{k} \lambda_{j}$, $k=2, \ldots, n$, and define $b_{j}$ by the recursive relation

$$
\begin{aligned}
b_{1} & =(1-\varepsilon)^{1 / 2}, \\
b_{j+1} & =\left(1-\lambda_{j+1}^{-1}\left(1-b_{j}^{2}\right)\right)^{1 / 2}, \quad j=1, \ldots, n .
\end{aligned}
$$


An easy induction argument shows that, with our choice of $\varepsilon$ we have $1-b_{j}^{2}<\lambda_{j+1}$. Therefore, formula (5.8) indeed defines a sequence of positive weights $b_{j}$. Also (5.8) implies the first $n-1$ equalities in (5.7); the last equality in $(5.7)$ holds because $1-b_{n}^{2}=\left(\lambda_{2} \cdots \lambda_{n}\right)^{-1}\left(1-b_{1}^{2}\right)$ and $\lambda_{1} \cdots \lambda_{n}=1$.

Thus, we have found a solution $B$ of (5.6). Letting $A=\Lambda^{1 / 2} B$, we get the two factorizations (5.5) and (5.6).

It remains to show that the eigenvalues of $\Lambda^{ \pm 1 / 2} B$ are all located in $\mathbf{D}_{+}$. To this end, notice that $\Lambda^{ \pm 1 / 2} B$ are weighted shifts simultaneously with $B$ and that

$$
\operatorname{det}\left(\Lambda^{ \pm 1 / 2} B\right)=\operatorname{det} B=(-1)^{n-1} b_{1} \cdots b_{n}
$$

Therefore, the eigenvalues of $\Lambda^{ \pm 1 / 2} B$ lie on the circle centered at the origin and having radius $r=\left(b_{1} \cdots b_{n}\right)^{1 / n}$. But, according to (5.8), all $b_{j}$ are smaller than 1 . Hence, $r<1$ as well.

Proof of Theorem 1.6. Pick any $k \in\{-(n-1), \ldots, n-1\}$. Let $J_{1}=J_{2}$ be the $n \times n$ Jordan block with the eigenvalue $e^{i \pi k / n}$. By Ballantine's Theorem 4.3, there are four positive definite matrices $H_{1}, H_{2}, H_{3}, H_{4}$ such that

$$
J_{1} J_{2}=H_{1}^{-1} H_{2} H_{3}^{-1} H_{4}
$$

Clearly,

$$
1=\operatorname{det}\left(J_{1} J_{2}\right)=\operatorname{det} H_{1}^{-1} \operatorname{det} H_{2} \operatorname{det} H_{3}^{-1} \operatorname{det} H_{4}
$$

This implies that we can find $\alpha>0$ and $\beta>0$ such that

$$
\operatorname{det}\left(\alpha H_{1}\right)=\operatorname{det}\left(\beta H_{4}\right), \quad \operatorname{det}\left(\alpha H_{2}\right)=\operatorname{det}\left(\beta H_{3}\right) \text {. }
$$

Put $D_{1}=\alpha H_{1}, D_{2}=\alpha H_{2}, D_{3}=\beta H_{3}, D_{4}=\beta H_{4}$. We then have

$$
J_{1} J_{2}=D_{1}^{-1} D_{2} D_{3}^{-1} D_{4}, \quad \operatorname{det} D_{1}=\operatorname{det} D_{4}, \quad \operatorname{det} D_{2}=\operatorname{det} D_{3},
$$

and letting $X:=D_{3}^{-1} D_{4} J_{2}^{-1}$, we therefore get

$$
J_{1}=D_{1}^{-1} D_{2} X, \quad J_{2}=X^{-1} D_{3}^{-1} D_{4}
$$


As the matrices $D_{1}, D_{2}, D_{3}, D_{4}$ are positive definite, we deduce from the last two equalities of (5.9) and from Lemma 5.3 that there are $2 \pi$-periodic $F_{l}, F_{r} \in A P W_{n \times n}$ such that

$$
\mathbf{d}_{r}\left(F_{l}\right)=D_{1}, \quad \mathbf{d}_{l}\left(F_{l}\right)=D_{4}, \quad \mathbf{d}_{l}\left(F_{r}\right)=D_{2}, \quad \mathbf{d}_{r}\left(F_{r}\right)=D_{3} .
$$

Put $A_{l}=F_{l}, A_{r}=F_{r} X$, choose any $A_{0} \in[C(\dot{\mathbf{R}})]_{n \times n}$ such that $A_{0}(\infty)$ is the zero matrix, and consider

$$
A(x)=(1-u(x)) A_{l}(x)+u(x) A_{r}(x)+A_{0}(x) .
$$

It is easily seen that $A_{0}$ can be chosen so that $\operatorname{det} A(x)$ is nonzero for all $x \in \mathbf{R}$. By construction and by (5.10),

$$
\begin{aligned}
& \left(\mathbf{d}_{r}\left(A_{l}\right)\right)^{-1} \mathbf{d}_{r}\left(A_{r}\right)=D_{1}^{-1} D_{2} X=J_{1} \\
& \left(\mathbf{d}_{r}\left(\tilde{A}_{l}\right)\right)^{-1} \mathbf{d}_{r}\left(\tilde{A}_{r}\right)=\left(\mathbf{d}_{l}\left(A_{r}\right)\right)^{-1} \mathbf{d}_{l}\left(A_{l}\right)=X^{-1} D_{3}^{-1} D_{4}=J_{2} .
\end{aligned}
$$

The eigenvalues $\xi_{j}$ of $J_{1}$ and $\tilde{\xi}_{j}$ of $J_{2}$ are all equal to $e^{i \pi k / n}$. Thus, Theorem 5.2 implies that $W(A)$ and $W(\tilde{A})$ are Fredholm and that

$$
\text { Ind } \begin{aligned}
W(A)+\operatorname{Ind} W(\tilde{A})= & -\frac{1}{2 \pi}\{\arg \operatorname{det} A\}_{-\infty}^{\infty}+\frac{1}{2 \pi} \sum_{j=1}^{n} \arg \xi_{j} \\
& +\frac{1}{2 \pi}\{\arg \operatorname{det} A\}_{-\infty}^{\infty}+\frac{1}{2 \pi} \sum_{j=1}^{n} \arg \tilde{\xi}_{j} \\
= & \frac{1}{2 \pi} n \cdot \pi \frac{k}{n}+\frac{1}{2 \pi} n \cdot \pi \frac{k}{n}=k .
\end{aligned}
$$

Consequently, Ind $W(A)+\operatorname{Ind} W(\tilde{A})$ may assume any prescribed value $k$ between $-(n-1)$ and $n-1$. On replacing $A(x)$ by

$$
A(x) \operatorname{diag}\left(\left(\frac{x-i}{x+i}\right)^{\mu}, 1, \ldots, 1\right)
$$

with appropriate integers $\mu$ we can finally obtain $A$ in $S A P_{n \times n}$, and even in $S A P W_{n \times n}$, such that Ind $W(A)$ and Ind $W(\tilde{A})$ are any integers $\kappa$ and $\nu$ subject to the constraint $|\kappa+\nu| \leq n-1$.

Acknowledgments. The research of Böttcher and Grudsky into this topic was essentially supported by DFG-Kooperationsprojekt 436 
RUS 113/426 for German and Russian scientists within the "Memorandum of Understanding" between DFG and RFFI. Böttcher and Spitkovsky acknowledge support by NATO Collaborative Research Grant CRG 950332. Grudsky also acknowledges support by RFFI Grant 98-01-01023, and Spitkovsky also acknowledges support by NSF Grant 9800704.

\section{REFERENCES}

1. C. Ballantine, Products of positive definite matrices III, J. Algebra 10 (1968), $174-182$.

2. — Products of positive definite matrices IV, Linear Algebra Appl. 3 (1970), 79-114.

3. A. Böttcher and S.M. Grudsky, Toeplitz operators with discontinuous symbols: phenomena beyond piecewise continuity, Oper. Theory Adv. Appl. 90 (1996), $55-118$.

4. A. Böttcher and B. Silbermann, Analysis of Toeplitz operators, Springer, Berlin, New York, 1990.

5. K. Clancey and I. Gohberg, Factorization of matrix functions and singular integral operators, Birkhäuser, Basel, 1981.

6. I. Gohberg and I.A. Feldman, Convolution equations and projection methods for their solutions, Transl. Math. Monographs 41, Amer. Math. Soc., Providence, R.I., 1974.

7. I. Gohberg and M.G. Krein, Systems of integral equations on a half-line with kernels depending on the difference of the arguments, Amer. Math. Soc. Transl. Ser. 214 (1960), 217-287.

8. R. Horn and C. Johnson, Topics in matrix analysis, Cambridge University Press, 1986.

9. Yu.I. Karlovich, On the Haseman problem, Demonstratio Math. 26 (1993), 581-595.

10. Yu.I. Karlovich and I.M. Spitkovsky, Factorization of almost periodic matrixvalued functions and the Noether theory for certain classes of equations of convolution type, Math. USSR-Izv. 34 (1990), 281-316.

11. G.S. Litvinchuk and I.M. Spitkovsky, Factorization of measurable matrix functions, Birkhäuser, Basel, 1987.

12. D. Sarason, Toeplitz operators with semi-almost periodic symbols, Duke Math. J. 44 (1977), 357-364.

13. I.B. Simonenko, The Riemann boundary value problem with measurable coefficients, Dokl. Akad. Nauk SSSR 135 (1960), 538-541, in Russian.

14. - The Riemann boundary value problem for $n$ pairs of functions with measurable coefficients and its application to the investigation of singular integrals in the spaces $L^{p}$ with weight, Izv. Akad. Nauk SSSR Ser. Mat. 28 (1964), 277-306, in Russian. 
15. Some general questions of the theory of the Riemann boundary value problem, Math. USSR-Izv. 2 (1968), 1091-1099.

16. I.M. Spitkovsky, On multipliers having no effect on factorability, Soviet Math. Dokl. 17 (1976), 1733-1738.

17. - On the factorization of measurable matrix functions in the classes $L^{p}(\varrho)$ with power weight, Izv. Vyssh. Uchebn. Zaved. Mat. 5 (1988), 62-70, in Russian.

18. H. Widom, Asymptotic behavior of block Toeplitz matrices and determinants II, Adv. Math. 21 (1976), 1-29.

Fakultät für Mathematik, TU Chemnitz, 09107 Chemnitz, Germany

E-mail address: aboettch@mathematik.tu-chemnitz.de

Faculty of Mechanics and Mathematics, Rostov-on-Don State University, Bolshaya Sadovaya 105, 344711 Rostov-on-Don, Russian Federation E-mail address: grudsky@aaanet.ru

Department of Mathematics, The College of William and Mary, WilliamsBURG, VA 23187-8795, USA

E-mail address: ilya@math.wm.edu 